\title{
Biclique Edge Cover Graphs and Confluent Drawings
}

\author{
Michael Hirsch, Henk Meijer, and David Rappaport \\ School of Computing, Queen's University \\ Kingston, Ontario, Canada
}

\begin{abstract}
Confluent drawing is a technique that allows some non-planar graphs to be visualized in a planar way. This approach merges edges together, drawing groups of them as single tracks, similar to train tracks. In the general case, producing confluent drawings automatically has proven quite difficult. We introduce the biclique edge cover graph that represents a graph $G$ as an interconnected set of cliques and bicliques. We do this in such a way as to permit a straightforward transformation to a confluent drawing of $G$. Our result is a new sufficient condition for confluent planarity and an additional algorithmic approach for generating confluent drawings. We give some experimental results gauging the performance of existing confluent drawing heuristics.
\end{abstract}

\section{Introduction}

In 2003, Dickerson, Eppstein, Goodrich, and Meng introduced confluent drawing, and with it a heuristic able to generate confluent drawings for some graphs [1]. These drawings present a novel way of visualizing non-planar graphs in a planar way, however, producing a planar confluent drawing for an arbitrary graph has proven to be quite difficult. Devine speculates that merely deciding whether such a drawing exists is NP-hard for an arbitrary graph 1]. Hui, Schaefer, and Stefankovic also speculate that this problem is NP-complete [2]. In this paper we explore alternate methods of automatically generating confluent drawings. We experimentally evaluate Dickerson et al.'s confluent drawing heuristic, as well as our own heuristics based on the biclique edge cover graph.

Francis Newbery proposed a method of merging together edges called edge concentration in a 1989 paper [3]. Dickerson et al. first introduced confluent drawings in [3] they have been subsequently studied in [2/415].

This paper is organized as follows. Section 2 provides a brief background, Sect. 3 defines the biclique edge cover graph, and Sect. 4 gives a method to transform such a graph into a confluent drawing. Finally, Sect. 5 covers confluent drawing algorithm implementations and their experimental performance.

\section{Background}

We define the relevant concepts in confluent drawing: A curve is a continuous map into the plane. A curve is smooth if it is continuously differentiable along 
its length (there are no sharp bends) [62]. A drawing $D$ is a confluent drawing of an undirected graph $G$ if:

- There is a one to one mapping between vertices of $G$ and $D$.

- There exists a smooth curve between vertices $u$ and $w$ in $D$ if and only if there exists an edge $(u, w)$ in $E$.

Consistent with [4] and [1, we have omitted the planarity constraint found in Dickerson et al.'s definition [6]. We say that a confluent drawing is planar if no smooth curve(s) intersect at a single point (they may share overlapping portions). A confluent drawing is non-planar if any curve(s) intersect at a single point.

Lastly, we define a switch, and traffic circle, two basic confluent elements. A switch is the point where curves converge. A switch $s$ is defined to have degree three [21], but we generalize it to have arbitrary degree. A traffic circle is a particular confluent representation of a clique such that all smooth curves merge with a central circular track. Figure 1 depicts a switch (left) and a traffic circle (right).
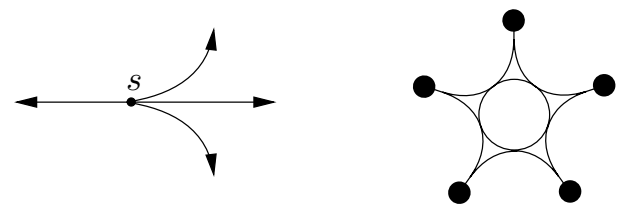

Fig. 1. A switch of degree four (left), and a confluent drawing of $K_{5}$ called a traffic circle (right)

\section{Biclique Edge Cover Graph}

Let $G=(V, E)$ be a graph. A clique $c$ is a subset of $V$ such that the subgraph induced by $c$ is a complete graph. We say that edge $e$ is in clique $c$ if it is in the subgraph induced by the vertices in $c$.

A biclique $\left(b_{i}, b_{j}\right)$ is an unordered pair of disjoint subsets $b_{i}$ and $b_{j}$ of $V$, such that for all $u \in b_{i}$ and $w \in b_{j},(u, w) \in E$. We call each subset $b_{i}$ and $b_{j}$ a $b$-part. We say that edge $e$ is in biclique $\left(b_{i}, b_{j}\right)$ if it is incident to a vertex in each b-part.

Let $C$ be a set of cliques, and let $B$ be a set of bicliques such that each edge of $G$ is in a clique of $C$ or in a biclique of $B$. We say that such sets $B$ and $C$ together edge cover $G$. Given a set of bicliques $B, B_{p}$ is the set of b-parts such that for each $\left(b_{i}, b_{j}\right) \in B, b_{i}, b_{j} \in B_{p}$.

Let $G$ be a graph. Let $B$ be a set of bicliques and let $C=\left\{c_{0}, c_{1}, \ldots, c_{m-1}\right\}$ be a set of cliques that together edge cover $G$. Let b-parts $b_{0}, b_{1}, \ldots, b_{l-1}$ denote the elements of $B_{p}$. Let $\pi_{0}, \pi_{1}, \ldots, \pi_{2^{l+m}-1}$ denote the elements in the power set of $B_{p} \cup C$. We define the biclique edge cover graph $G_{b}=\left(V_{b}, E_{b}\right)$ as follows: 
- Vertex $v_{b}=\pi_{i}$ is in $V_{b}$ if and only if there exists a vertex $v \in V$ such that $v$ is in every b-part and clique in $\pi_{i}$ and no others.

- Edge $e_{b}=\left(u_{b}, w_{b}\right)$ is in $E_{b}$ if and only if $u_{b} \neq w_{b}$, and $u_{b} \cap w_{b} \cap C \neq \emptyset$, or there exists a $b_{i} \in u_{b}$ and $b_{j} \in w_{b}$ such that $\left(b_{i}, b_{j}\right) \in B$.

We say that $v$ and $v_{b}$ are associated if a vertex $v \in V$ is in every b-part and clique in $v_{b}=\pi_{j} \in V_{b}$ and no others. Hereafter, $u, v, w$ and $v_{0}, v_{1}, \ldots, v_{n-1}$ denote the elements of $V$. Similarly, $u_{b}, v_{b}, w_{b}$ and $v_{b_{0}}, v_{b_{1}}, \ldots, v_{b_{n-1}}$ denote the elements of $V_{b}$.

\subsection{Example}

The following example illustrates the derivation of a biclique edge cover graph $G_{b}$ from graph $G$ (Fig. 2). Given a graph $G$, first determine a set of bicliques $B$, and cliques $C$. Note that any sets will suffice, provided that $B$ and $C$ together edge cover $G$. We choose $B=\left\{\left(\left\{v_{0}, v_{1}\right\},\left\{v_{2}, v_{3}, v_{4}\right\}\right)\right\}$ and $C=\left\{c_{0}\right\}=$ $\left\{v_{2}, v_{3}, v_{4}, v_{5}, v_{6}\right\}$. Thus $B_{p}=\left\{b_{0}, b_{1}\right\}=\left\{\left\{v_{0}, v_{1}\right\},\left\{v_{2}, v_{3}, v_{4}\right\}\right\}$.

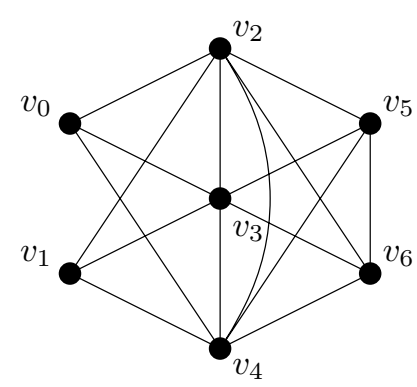

Fig. 2. Graph $G$

We construct the vertex set $V_{b}$ : The vertex $v_{0} \in b_{0}, v_{0} \notin b_{1} \cap c_{0}$. By our definition, $v_{0}$ establishes $\left\{b_{0}\right\} \in V_{b}$, and $v_{0}$ and $\left\{b_{0}\right\}$ are associated. Vertex $v_{1}$ also establishes $\left\{b_{0}\right\} \in V_{b}$. Vertices $v_{2}, v_{3}, v_{4}$ each establish $\left\{b_{1}, c_{0}\right\} \in V_{b}$. Vertices $v_{5}$ and $v_{6}$ establish $\left\{c_{0}\right\} \in V_{b}$. Thus $V_{b}=\left\{v_{b_{0}}, v_{b_{1}}, v_{b_{2}}\right\}=\left\{\left\{b_{0}\right\},\left\{b_{1}, c_{0}\right\},\left\{c_{0}\right\}\right\}$. The edge set $E_{b}=\left\{\left(v_{b_{1}}, v_{b_{2}}\right),\left(v_{b_{0}}, v_{b_{1}}\right)\right\}$. Figure 3 depicts biclique edge cover graph $G_{b}$. We use a solid vertex to depict any $v_{b} \cap C \neq \emptyset$, and an unfilled vertex to depict any $v_{b} \cap C=\emptyset$.

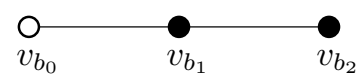

Fig. 3. Derived biclique edge cover graph $G_{b}$ 
Lemma 1. Let $G$ be a graph. Let $B$ be a set of bicliques and let $C$ be a set of cliques that together edge cover $G$. Let $G_{b}$ be the resulting biclique edge cover graph. The following properties hold:

1. The magnitude $\left|V_{b}\right| \leq|V|$.

2. Vertices $v_{0}, v_{1}, \ldots, v_{r-1} \in V$ associated with a vertex $v_{b} \in V_{b}$ define an independent set in $G$ where $v_{b} \cap C=\emptyset$; otherwise, they define a clique in $G$.

Proof. Property (1) follows from our definition of a biclique edge cover graph: Each vertex $v \in V$ may only be associated with a single vertex $v_{b} \in V_{b}$. Each vertex $v_{b} \in V_{b}$ is associated with at least one vertex $v \in V$. It follows that $\left|V_{b}\right| \leq|V|$.

We prove Property (2) by contradiction. Let $u$ and $w$ be adjacent vertices in $V$, such that $u$ and $w$ are associated with $v_{b} \in V_{b}$ and $v_{b} \cap C=\emptyset$. By definition, edge $e=(u, w)$ is in a biclique in $B$ or a clique in $C$. If $e$ is in a clique $c \in C$, then $c$ must be an element of $v_{b}$. This is contrary to $v_{b} \cap C=\emptyset$. If $e$ is in a biclique $\left(b_{i}, b_{j}\right) \in B$, it follows that $u$ must be an element of $b_{i}$ and $w$ an element of $b_{j}$. Recall that b-parts $b_{i}$ and $b_{j}$ are disjoint. Vertices $u$ and $w$ cannot therefore both be associated with $v_{b}$. Where $v_{b} \cap C \neq \emptyset$, Property (2) follows directly from the definition.

\section{Generating Confluent Drawings}

In this section we show how to construct a drawing $D$ of $G$ from a drawing of its biclique edge cover graph $G_{b}$. Let $G$ be a graph. Let $B$ be a set of bicliques and let $C$ be a set of cliques that together edge cover $G$. Let $G_{b}$ be the resulting biclique edge cover graph. Let $D_{b}$ be a drawing of $G_{b}$. Note that drawing $D_{b}$ could be a traditional drawing or a confluent drawing of $G_{b}$. Replace each vertex $v_{b_{i}} \in D_{b}$ by vertices of $G$ as follows:

We will compose a confluent structure. Begin with a single circular track. Join each vertex in $V$ associated with $v_{b_{i}}$ to the circular track:

- If $v_{b_{i}} \cap C \neq \emptyset$ : Join each vertex in $V$ associated with $v_{b_{i}}$ to the circular track by means of two smooth curves such that one curve may be followed onto, and around the adjoined circular track in the clockwise direction, and the other in the counterclockwise direction. We call this construction a traffic circle.

- If $v_{b_{i}} \cap C=\emptyset$ : Join each vertex in $V$ associated with $v_{b_{i}}$ to the circular track by means of a smooth curve such that the curve may be followed onto, and around the adjoined circular track in the counterclockwise direction. We call this construction a counterclockwise traffic circle.

Remove vertex $v_{b_{i}}$ from drawing $D_{b}$, and put the composed confluent structure in its place. Merge all (confluent) edges previously incident to $v_{b_{i}}$ with the circular track such that each edge may be followed onto, and around the adjoined 
circular track in the clockwise direction. Figure 4 illustrates our replacement method for a vertex $v_{b_{i}} \in D_{b}$ with two incident edges, and three associated vertices in $V$.

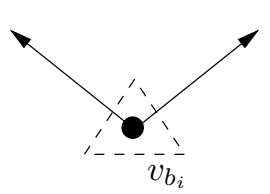

(a)

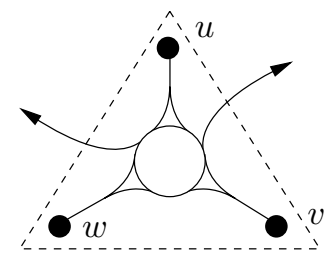

(b)

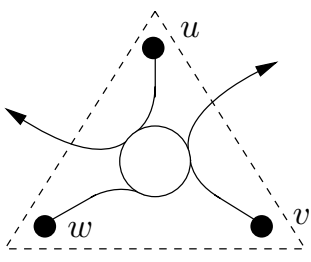

(c)

Fig. 4. (a) Vertex $v_{b_{i}}$ in drawing $D_{b}$ (b) Replacement of $v_{b_{i}}$ in drawing $D$ where $v_{b_{i}} \cap C \neq \emptyset$ (c) Replacement of $v_{b_{i}}$ in drawing $D$ where $v_{b_{i}} \cap C=\emptyset$

\subsection{Example}

We continue with the example of Sect. 3.1. We generate a confluent drawing of $G$ from a drawing of $G_{b}$ (Fig. 3). Each vertex of $G_{b}$ is replaced by all associated vertices of $G$. The result is drawing $D$, Fig. 5 .

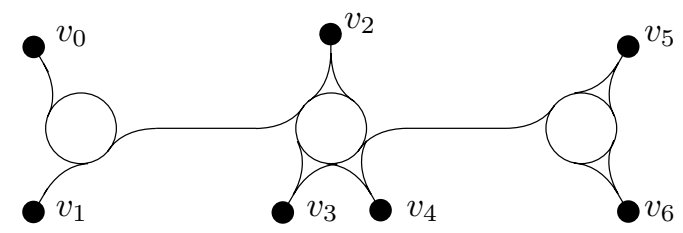

Fig. 5. Confluent drawing $D$ generated from a drawing of $G_{b}$

Lemma 2. Let $D$ be a drawing generated from a drawing of $G_{b}$ by the method of this section. Drawing $D$ is a confluent drawing of $G$.

Proof. We first show that a one to one mapping exists between vertices of $G$ and $D$. We then show that there exists a smooth curve between vertices $u$ and $w$ in $D$ if and only if there exists an edge $(u, w)$ in $E$. We present this argument in two cases.

Our method replaces each vertex $v_{b} \in V_{b}$ by all associated vertices in $V$. Because each vertex in $V$ is associated with a single vertex in $V_{b}$, the vertex set of $D$ is precisely that of $G$.

Case $I$. We will show that there exists a smooth curve between vertices $u$ and $w$ in $D$ if there exists an edge $(u, w)$ in $E$. Vertices $u$ and $w$ are either associated with the same vertex, or two different vertices in $V_{b}$. If they are associated with 
the same vertex $v_{b_{i}} \in V_{b}$ and $v_{b_{i}} \cap C \neq \emptyset$, then our method sees that $u$ and $w$ are in the same constructed traffic circle. A smooth curve therefore exists between $u$ and $w$ in $D$. Otherwise (if $v_{b_{i}} \cap C=\emptyset$ ) Lemma 1 precludes $(u, w)$ from being an edge in $E$.

We now show that a smooth curve exists between $u$ and $w$ if they are associated with different vertices in $V_{b}: u$ with $u_{b}$ and $w$ with $w_{b}$. By definition, edge $(u, w)$ is in a biclique in $B$ or a clique in $C$. If $(u, w)$ is in a clique $c \in C$, then clique $c$ is an element of both $u_{b}$ and $w_{b}$. Vertices $u_{b}, w_{b}$ are therefore adjacent. If $(u, w)$ is in a biclique $\left(b_{i}, b_{j}\right) \in B$, then $b_{i}$ is an element of $u_{b}$ and $b_{j}$ an element of $w_{b}$. Vertices $u_{b}, w_{b}$ are again adjacent. Our method ensures that the (confluent) edge between $u_{b}$ and $w_{b}$ is merged with the circular track that replaces $u_{b}$ and the circular track that replaces $w_{b}$. This merged edge completes a smooth curve between $u$ and $w$ in $D$.

Case II. We will show that there exists an edge $(u, w)$ in $E$ if there exists a smooth curve between vertices $u$ and $w$ in $D$. Our method generates smooth curves in $D$ in two ways. First, constructed traffic circles consist of smooth curves between vertices of $V$. Two vertices $u$ and $w \in V$ are in the same traffic circle only if they are associated with the same vertex in $V_{b}$. It follows from Lemma 1 that edge $(u, w) \in E$.

Additionally, smooth curves connect traffic circles/circular tracks that have replaced adjacent vertices in $V_{b}$. Because (confluent) edges are always merged with these confluent structures in the same direction, a smooth curve never connects two structures that have replaced non-adjacent vertices in $V_{b}$. Smooth curves in $D$ therefore connect vertices $u$ and $w$ that are associated with adjacent vertices in $V_{b}$. If vertices $u_{b}, w_{b} \in V_{b}$ are adjacent, then there exists a clique $c \in u_{b} \cap w_{b}$ or there exist b-parts $b_{i} \in u_{b}$ and $b_{j} \in w_{b}$ such that $\left(b_{i}, b_{j}\right) \in B$. If $c \in u_{b} \cap w_{b}$, then any vertex associated with either $u_{b}$ or $w_{b}$ is in $c$. Otherwise, if $b_{i} \in u_{b}$ and $b_{j} \in w_{b}$, then $u$ is an element of $b_{i}$ and $w$ an element of $b_{j}$. In either case, it follows that edge $(u, w) \in E$.

\subsection{Planarity}

Lemma 3. Let $D$ be a drawing generated from a drawing of $G_{b}$ by the method of this section. If the drawing of $G_{b}$ is confluent planar then $D$ is confluent planar.

Proof. Our method replaces vertices in the drawing of $G_{b}$ by confluent planar structures to produce $D$. No edge crossings exist in the drawing of $G_{b}$, and none are introduced by our replacement scheme. Drawing $D$ is therefore a confluent planar drawing of $G$.

Corollary 1. If the drawing of $G_{b}$ is planar then $D$ is confluent planar.

Proof. A planar graph satisfies the definition of confluent planarity.

\subsection{Prickly Clique}

A prickly clique consists of a clique and one additional vertex adjacent to each vertex in the clique. More formally, a prickly clique is a graph $G$ on $2 n$ vertices 


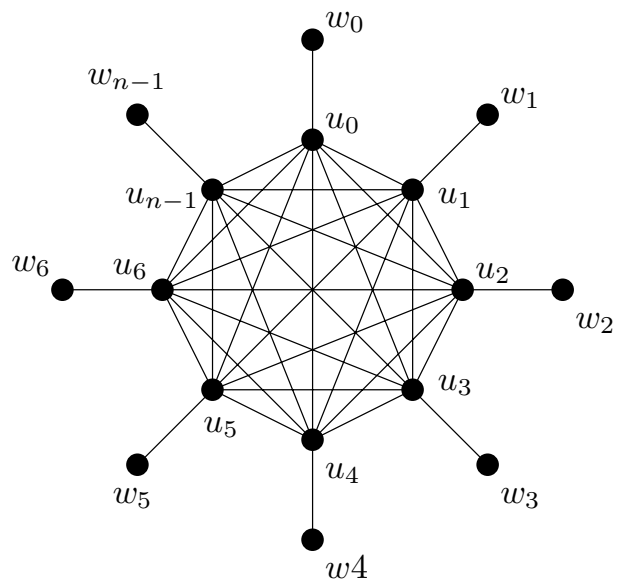

Fig. 6. Prickly clique $G$ for $n=8$

with $n \geq 2$ such that $V=\left\{u_{0}, u_{1}, \ldots u_{n-1}, w_{0}, w_{1}, \ldots, w_{n-1}\right\}$, and $E=E_{0} \cup E_{1}$ where $\left(u_{i}, w_{i}\right) \in E_{0}$ for $0 \leq i<n$ and $\left(u_{i}, u_{j}\right) \in E_{1}$ for $0 \leq i<j<n$. The prickly clique for $n \geq 5$ is an example of a confluent planar graph that does not have a resulting planar biclique edge cover graph.

Lemma 4. Let $G$ be a prickly clique. Let $B$ be a set of bicliques and let $C$ be a set of cliques that together edge cover $G$. Let $G_{b}$ be the resulting biclique edge cover graph. Graph $G$ is isomorphic to $G_{b}$.

Proof. We construct the vertex set $V_{b}$. Each edge $\left(u_{i}, w_{i}\right) \in E_{0}$ is either a clique in $C$ or a biclique in $B$, while each edge $\left(u_{i}, u_{j}\right) \in E_{1}$ is in at least one biclique in $b$ or clique in $C$. Thus, each vertex $w_{i} \in G$ establishes a vertex $w_{b_{i}} \in V_{b}$, while each vertex $u_{i} \in G$ establishes a vertex $u_{b_{i}} \in V_{b}$. This defines a bijection $u_{i} \rightarrow u_{b_{i}}, w_{i} \rightarrow w_{b_{i}}$, from $V$ to $V_{b}$.

We construct the edge set $E_{b}$. Edge $\left(u_{b_{i}}, w_{b_{i}}\right) \in E_{b}$ for $0 \leq i<n$ (if $\left\{u_{i}, w_{i}\right\} \in$ $C$ then $u_{b_{i}} \cap w_{b_{i}} \cap\left\{u_{i}, w_{i}\right\} \neq \emptyset$; otherwise if $\left\{\left\{u_{i}\right\},\left\{w_{i}\right\}\right\} \in B$ then $\left\{u_{i}\right\} \in u_{b_{i}}$ and $\left.\left\{w_{i}\right\} \in w_{b_{i}}\right)$. Moreover, edge $\left(u_{b_{i}}, u_{b_{j}}\right) \in E_{b}$ for $0 \leq i<j<n$ (if $\left(u_{i}, u_{j}\right)$ is in a clique $c \in C$ then $u_{b_{i}} \cap u_{b_{j}} \cap c \neq \emptyset$; otherwise if $\left(u_{i}, u_{j}\right)$ is in a biclique $b=\left(b_{i}, b_{j}\right) \in B$ then $b_{i} \in u_{b_{i}}$ and $\left.b_{j} \in u_{b_{j}}\right)$.

\section{Implementation and Results}

In this section we examine two algorithmic approaches for generating confluent drawings. We will examine the experimental performance of each implemented algorithm, and conclude with some sample outputs. 


\subsection{ConfluentDickerson $(G)$}

Algorithm 1 is based on the only previously published confluent drawing algorithm for undirected graphs. Presented by Matthew Dickerson at the 11th International Symposium on Graph Drawing, the heuristic iteratively identifies and replaces large cliques and bicliques (complete and bipartite subgraphs) with equivalent confluent structures [6].

Our implementation uses an $O(n m \mu)$ solution (where $\mu$ is the number of maximal independent sets of a graph) to the maximal independent sets problem by Tsukiyama et al. 7] to enumerate all maximal cliques and bicliques. This solution yields all maximal cliques when applied to a graph's complement, and all maximal bicliques when applied to its double cover [8]. Note that Dickerson et al. 6] specified an algorithm by Chiba and Nishizeki 9] for identifying cliques and a second algorithm by Eppstein [10] for identifying bicliques.

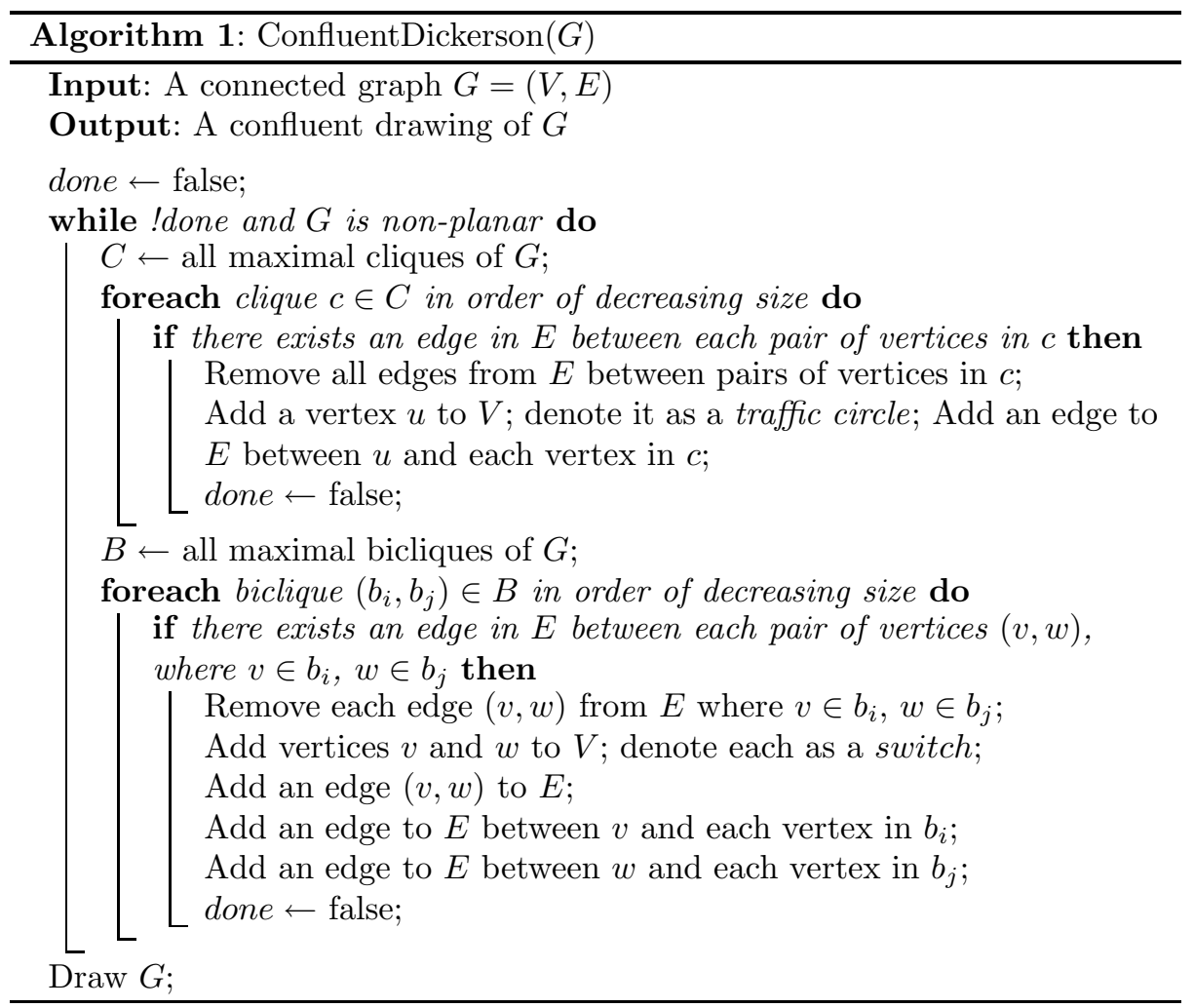

\subsection{ConfluentHirsch $(G)$}

Algorithm 2 is an implementation of the algorithm presented in Secs. 3 and 4. The algorithm first randomly computes a set of cliques and bicliques that 
together edge cover $G$. It then determines the vertex set of the biclique edge cover graph. Each of these vertices is inserted into $G$, joined to all associated vertices, and denoted as a traffic circle or counterclockwise traffic circle.

RecursiveHirsch $(G, i)$. Beginning with $G$, this variation of ConfluentHirsch $(G)$ iteratively computes $i$ successive biclique edge cover graphs. A confluent drawing of $G$ is recursively constructed using the algorithm in Sec 4] See [11] for details.

$\operatorname{DiscardHirsch}(G)$. This second variation discards cliques $|c| \leq 3$ from $C$ and bicliques $\left|b_{i}\right|,\left|b_{j}\right| \leq 1$ from $B$. Intuitively, including these degenerate cases can only hamper the performance of our algorithm. Any vertices that are no longer in a clique or biclique after the discard are effectively ignored by the algorithm.

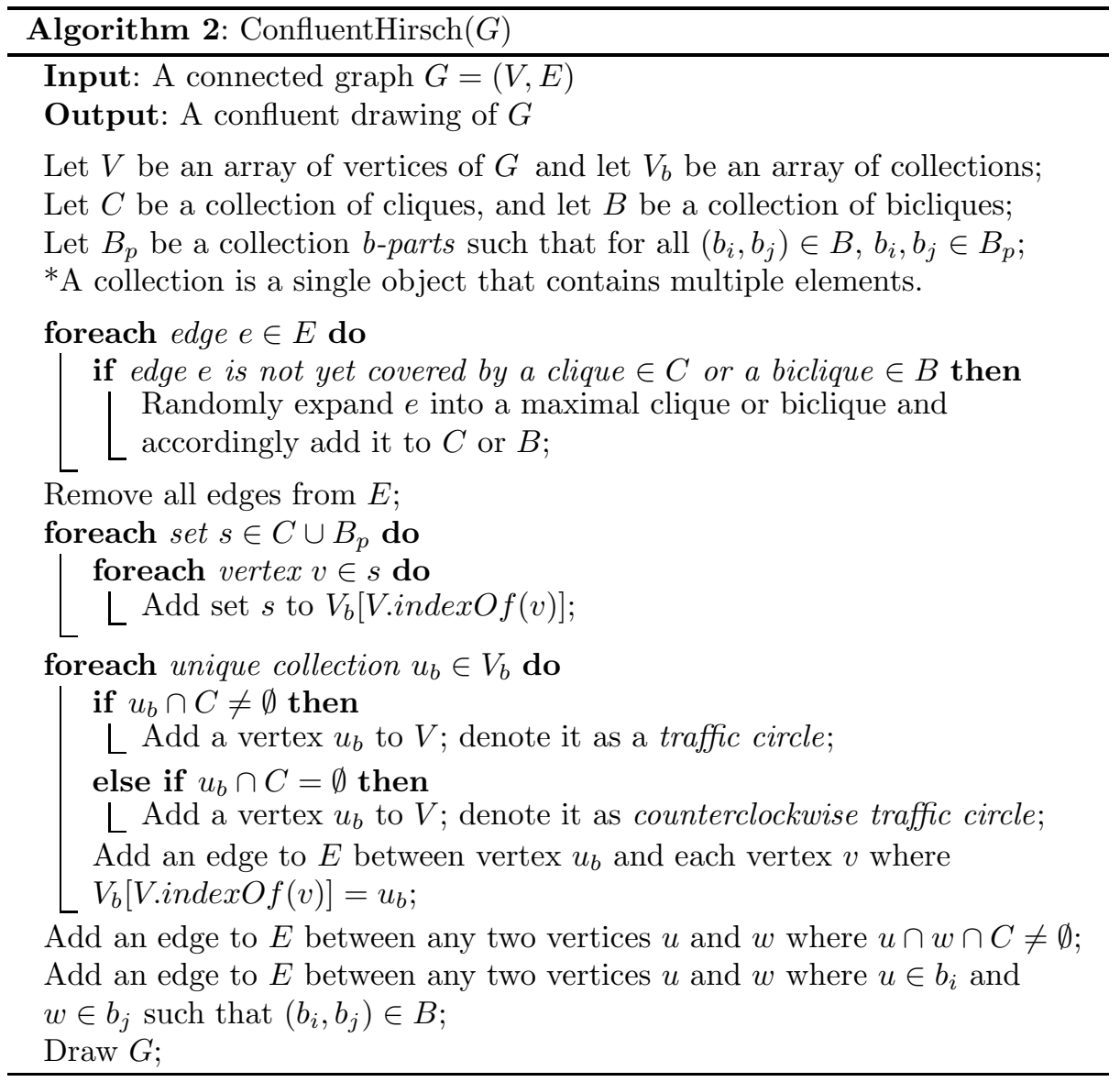




\subsection{Experimental Results}

We have applied our drawing algorithm implementations to two sets of graphs in order to measure their performance 1 . Table 1 summarizes results for the Rome graphs [12, and Table 2 for the ATT graphs (http://www.graphdrawing.org).

Because ConfluentHirsch $(G)$ and DiscardHirsch $(G)$ are non-deterministic, they were allowed multiple attempts per input to produce a confluent planar output. For a given case, a single confluent planar output was recorded if at least one attempt produced a confluent planar output. Note that RecursiveHirsch $(G, i)$ is also non-deterministic, however, multiple recursive iterations ensure that its output is not determined by one random set of cliques and bicliques.

Table 1. Performance of confluent drawing algorithms on the Rome set

\begin{tabular}{|c|c|c|c|c|}
\hline Algorithm applied & $\begin{array}{l}\text { Non-planar } \\
\text { inputs }\end{array}$ & $\begin{array}{l}\text { Attempts } \\
\text { per input }\end{array}$ & $\begin{array}{l}\text { Confluent } \\
\text { planar } \\
\text { outputs }\end{array}$ & $\begin{array}{l}\text { Confluent planar out- } \\
\text { puts not found by } \\
\text { ConfluentDickerson }(G)\end{array}$ \\
\hline ConfluentDickerson(G) & 8253 & 1 & 210 & - \\
\hline ConfluentHirsch $(\mathrm{G})$ & 8253 & 10 & 9 & 1 \\
\hline ConfluentHirsch(G) & 8253 & 100 & 10 & 1 \\
\hline RecursiveHirsch $(\mathrm{G}, 10)$ & 8253 & 1 & 10 & 1 \\
\hline RecursiveHirsch $(G, 100)$ & 8253 & 1 & 10 & 1 \\
\hline $\operatorname{DiscardHirsch}(G)$ & 8253 & 10 & 115 & 22 \\
\hline
\end{tabular}

Table 2. Performance of confluent drawing algorithms on the ATT set

\begin{tabular}{|c|c|c|c|c|}
\hline$\overline{\text { Algorithm applied }}$ & $\begin{array}{l}\text { Non-planar } \\
\text { inputs }\end{array}$ & $\begin{array}{l}\text { Attempts } \\
\text { per input }\end{array}$ & $\begin{array}{l}\text { Confluent } \\
\text { planar } \\
\text { outputs }\end{array}$ & $\begin{array}{l}\text { Confluent planar out- } \\
\text { puts not found by } \\
\text { ConfluentDickerson }(G)\end{array}$ \\
\hline ConfluentDickerson(G) & 423 & 1 & 166 & - \\
\hline ConfluentHirsch $(\mathrm{G})$ & 423 & 10 & 48 & 0 \\
\hline ConfluentHirsch(G) & 423 & 100 & 53 & 0 \\
\hline RecursiveHirsch $(\mathrm{G}, 10)$ & 423 & 1 & 57 & 1 \\
\hline RecursiveHirsch $(\mathrm{G}, 100)$ & 423 & 1 & 61 & 2 \\
\hline $\operatorname{DiscardHirsch}(G)$ & 423 & 10 & 129 & 5 \\
\hline
\end{tabular}

Figures 7 and 8 were output by our implementation 2 Switches are denoted $S$, with an arrowhead marking the incident edge along which the other incident edges converge. Traffic circles are denoted $C$ :

${ }^{1}$ Planarity was determined using the Lempel-Even-Cederbaum planarity test implementation included as part of GTL, the Graph Template Library (http://www. infosun.fmi.uni-passau.de/GTL).

2 Our implementation uses the Graphviz 13 . package to produce layouts and drawings, as well as the Grappa 14] package for working with graphs in Java. 


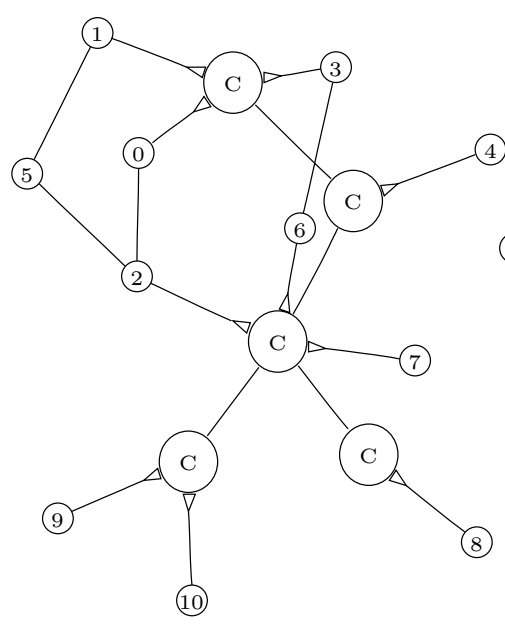

(a) DiscardHirsch $(G)$

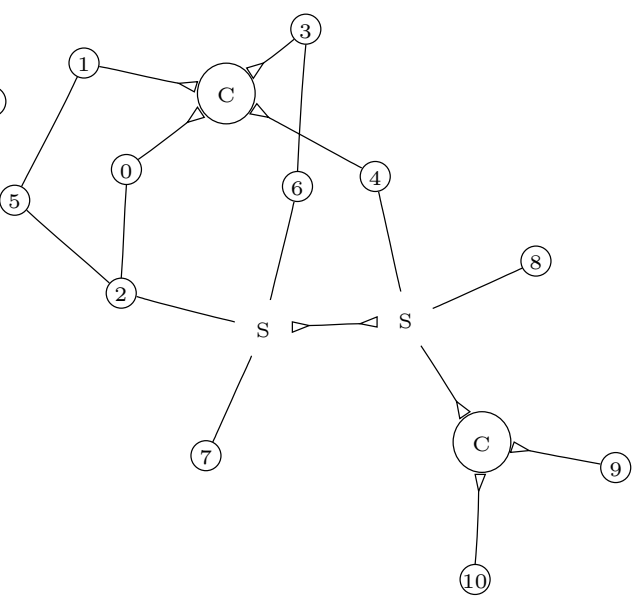

(b) ConfluentDickerson $(G)$

Fig. 7. Confluent drawings of an example given by Dickerson et al. in 6]. Spring embedder layout computed using Graphviz [13].

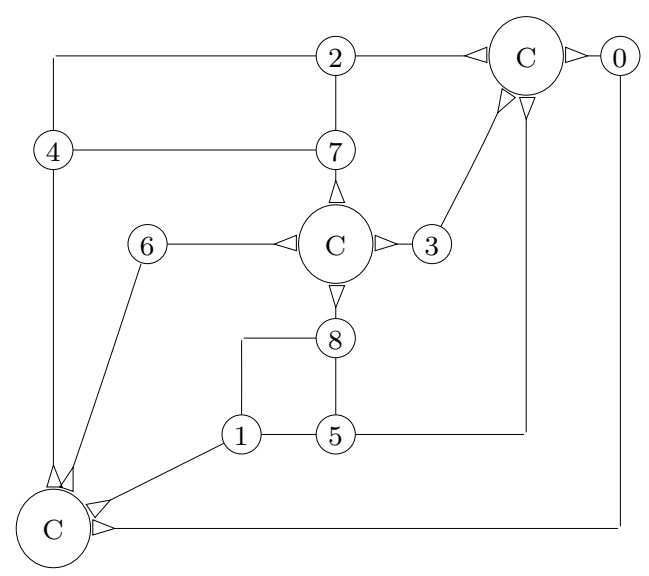

Fig. 8. The smallest known confluent non-planar graph is the Peterson graph with one vertex and adjacent edges removed [12. Above, a confluent planar drawing of its complement generated by ConfluentDickerson $(G)$. Layout computed using the dominancepolyline method for general undirected planar graphs in [15.

\section{Conclusion}

The performance of the algorithms varied, with ConfluentDickerson $(G)$ producing the greatest number of confluent planar drawings for both sets of graphs. 
Each variation of ConfluentHirsch $(G)$ was however able to produce confluent planar results for some inputs where ConfluentDickerson $(G)$ was not. Our results seem to confirm that confluent drawings offer a valid means for drawing nonplanar graphs in a planar way for some inputs. Confluent drawings can however be more difficult to read than traditional drawings. This holds true even for cases where a confluent drawing is planar and the original graph is not.

\section{References}

1. Devine, J.: Confluent graphs. Master's thesis, Queen's University (2005)

2. Hui, P., Schaefer, M., Štefankovič, D.: Train tracks and confluent drawings. In Pach, J., ed.: Proc. 12th Int. Symp. Graph Drawing (GD 2004). Number 3383 in Lecture Notes in Computer Science, Springer-Verlag (2004) 318-328

3. Newbery, F.J.: Edge concentration: a method for clustering directed graphs. In: Proc. 2nd Int. Works. on Soft. configuration management, ACM Press (1989) 76-85

4. Eppstein, D., Goodrich, M.T., Meng, J.Y.: Confluent layered drawings. In Pach, J., ed.: Proc. 12th Int. Symp. Graph Drawing (GD 2004). Number 3383 in Lecture Notes in Computer Science, Springer-Verlag (2004) 184-194

5. Eppstein, D., Goodrich, M.T., Meng, J.Y.: Delta-confluent drawings. In Healy, P., Nikolov, N.S., eds.: Proc. 13th Int. Symp. Graph Drawing (GD 2005). Number 3843 in Lecture Notes in Computer Science, Springer-Verlag (2006) 165-176

6. Dickerson, M.T., Eppstein, D., Goodrich, M.T., Meng, J.Y.: Confluent drawings: visualizing non-planar diagrams in a planar way. In: Proc. 11th Int. Symp. Graph Drawing (GD 2003). Lecture Notes in Computer Science, Springer-Verlag (2003)

7. Tsukiyama, S., Ide, M., Ariyoshi, H., Shirakawa, I.: A new algorithm for generating all the maximal independent sets. In: SIAM Journal on Computing. Volume 6. (1977) 505-517

8. Alexe, G., Alexe, S., Crama, Y., Foldes, S., Hammer, P.L., Simeone, B.: Consensus algorithms for the generation of all maximal bicliques. Discrete Applied Mathematics 145(1) (2004) 11-21

9. Chiba, N., Nishizeki, T.: Arboricity and subgraph listing algorithms. SIAM J. Comput. 14(1) (1985) 210-223

10. Eppstein, D.: Arboricity and bipartite subgraph listing algorithms. Information Processing Letters 51(4) (1994) 207-211

11. Hirsch, M.: Generating confluent drawings: Theory and practice. Master's thesis, Queen's University (2006)

12. Di Battista, G., Garg, A., Liotta, G.: An experimental comparison of three graph drawing algorithms (extended abstract). In: Proc. 11th Annual Symp. Computational Geometry (SCG '95), New York, NY, USA, ACM Press (1995) 306-315

13. Gansner, E.R., North, S.C.: An open graph visualization system and its applications to software engineering. Softw. Pract. Exper. 30(11) (2000) 1203-1233

14. Barghouti, N.S., Mocenigo, J., Lee, W.: Grappa: A graph package in java. In Di Battista, G., ed.: Proc. 5th Int. Symp. Graph Drawing (GD '97). Volume 1353 of Lecture Notes in Computer Science., Springer (1997) 336-343

15. Di Battista, G., Eades, P., Tamassia, R., Tollis, I.G.: Graph Drawing: Algorithms for the Visualization of Graphs. Prentice Hall, NJ, USA (1998) 